\title{
Oficinas educativas sobre o autocuidado de pacientes com diabetes mellitus em uma unidade de saúde atendida pelo "Programa Mais Médicos" no Brasil: relato de experiência.
}

\section{Educational workshops on self-care of patients with diabetes mellitus in a health facility served by "Programa Mais Médicos" in Brazil : experience report .}

Renato Penha de Oliveira SANTOS

Martha Leticia Carbonell VELAZQUE ${ }^{2}$

RESUMO: As doenças crônicas, como a Diabetes Mellitus, é um dos agravos à saúde que mais demandam por ações de educação em saúde, como o autocontrole dos níveis de glicose, a prática de atividade física e a dieta alimentar, sendo, estas, instrumentos fundamentais para o seu controle. Neste estudo, objetiva-serelatar a experiência de educação à saúde oferecida por meio de oficinas educativas para pessoas com diagnostico de Diabetes Mellitus e com dificuldades para alcançar a meta glicêmica em seu tratamento. Foi utilizada metodologia participativa e técnicas lúdicas, envolvendo a participação de 50 usuários com diabetes e cadastrados na Unidade Básica de Saúde de Ipuca,a qual é uma das unidades inscritas no Programa Mais Médicos, no município de São Fidelis, no período de dois anos.A metodologia participativa valorizou o conhecimento prévio dos participantes em relação à fisiopatologia, dieta e atividade física e a co-responsabilidades frente ao autocuidado. A oficina mostrou-se como espaço de reflexão e debate sobre a adoção de hábitos saudáveis, demonstrando queas vivências e as experiências em se trabalhar de forma conjunta profissionais de saúde e pacientes, auxiliam na redução da mortalidade por diabetes e na melhoria da qualidade de vida e o melhor cuidado do usuário e da comunidade.

Palavras-chave: Educação em Saúde; Diabetes Mellitus; Autocuidado; Atenção Primária à Saúde.

\footnotetext{
1 Médico de família e comunidade, mestrando na Escola Politécnica de Saúde Joaquim Venâncio e supervisor do Programa Mais Médicos para o Brasil, nos municípios de Cambuci e São Fidélis, no estado do Rio de Janeiro

2 Médica intercambista do projeto Mas Médicos para o Brasil, na UBS Jose de Abreu ,na comunidade de Ipuca, no município São Fidélis ,RJ.Especialista de primeiro e segundo grau em Medicina General Integral ,Professora Assistente, Msc em Atenção Integral nas Crianças
} 
SUMMARY: Chronic diseases, such as Diabetes Mellitus, is one of the harms to health are those that more demand for health education actions, like self-control glucose levels, the practice of physical activity and diet, being, these fundamental instruments for your control. In this study, the goal is reporting the health education experiencedeliveredthrough educational workshops for people with a diagnosis of Diabetes Mellitus and with difficulties to reach the goal Glycemic in your treatment. Methodology was used and playfultechniquesinitiativeparticipates, involving the participation of 50 users with diabetes and registered in Basic Health Unit of Ipuca, which is one of the unitslisted in the program more doctors, in the municipality of St. Fidelis. The participatory methodology apreciatted prior knowledge of participants in relation topathophysiology, diet and physical activity and responsibility in front of the self-care. The workshop has shownitself as a space for reflection and debate on the adoption of healthyhabits, demonstrating that the experiences and the experiences in workingtogether health professionals and patients, assist in reducing mortality from diabetes and improving the quality of life and better user and community care. Keywords: health education; Diabetes Mellitus; Self-care; Primary health care.

RESUMEN: Enfermedades crónicas como la Diabetes Mellitus es uno de los daños a la salud que más demandan de acciones de Educación de salud, el autocontrol en los niveles de glucosa, la práctica de actividad física y dieta, son, estos instrumentos fundamentales para su control. En este estudio, el objetivo es informar la experiencia de Educación de salud entregada a través de talleres educativos para personas con un diagnóstico de la Diabetes Mellitus y con dificultades para alcanzar el índice glicémico en el tratamiento. Se utilizó la metodología participativa con iniciativa de técnicas lúdicas, con la participación de 50 usuarios con diabetes y registrados en la unidad básica de salud de Ipuca, que es una de las unidades que figuran en el programa de más médicos, en el município de São Fidelis. La metodología participativa valorizo el conocimiento previo de los participantes en relación con la Fisiopatología, la dieta y la actividad física y responsabilidad frente a los cuidados personales. El taller se mostro como un espacio de reflexión y debate sobre la adopción de hábitos saludables, demostrando que las experiencias y las vivencias en el trabajo de profesionales de la salud y pacientes, ayudan a reducir la mortalidad por diabetes y a mejorar la calidad de vida y mejora el cuidado de pacientes y la atención comunitaria.

Palabras clave: Educación para la salud; Diabetes Mellitus; Cuidados personales; Atención primaria de salud.

\section{INTRODUÇÃO}

O diabetes mellitus (DM) é considerada pela Organização Mundial de Saúde (OMS) e pela Federação Internacional de Diabetes (IDF) como um problema de saúde pública. É uma síndrome de etiologia múltipla causada pela falta de insulina e/ou incapacidade da mesma de exercer adequadamente seus efeitos, "caracterizada por hiperglicemia crônica, frequentemente acompanhada de dislipidemia, hipertensão arterial e disfunção endotelial”. ${ }_{(1)}$ 
A Organização mundial da saúde (OMS) tem prognosticado que no ano 2030 o diabetes mellitus afetará a 370 milhões de pessoas e supõe um aumento de 11,4 \% com respeito nos últimos dados publicados em relação a esta doença. Segundo estudos realizados por Delgado, em 2009, esta doença se distribui por todo o mundo, "afetando 5,1 \% da população mundial (177 milhões de doentes) entre os quais, mais de 90 \% são pacientes com Diabetes Mellitus tipo 2 e as cifras globais de prevalência em adultos oscilam entre 4 e 7 \%, em relação ao sub registro no diagnostico ${ }_{(2)}$

A incidência de DM tipo 2 aumenta gradativamente com a idade sendo as pessoas entre 30 e 69 anos as mais afetadas. No Brasil a prevalência nesta faixa etária é de 7,6 \%, calcula - se que no ano 2025 possam existir 11 milhões de diabéticos no país. ${ }_{(3)}$

O DM representa a quarta causa de morte no Brasil, com altos níveis de morbilidade e mortalidade e considerada uma das mais importantes doenças endócrinas metabólicas em nosso meio. É estimado que o Brasil passe da oitava posição, a nível mundial, com prevalência de 4,6 \% no ano 2000, para a sexta posição com 11,3 \% no ano 2030, sendo os hábitos alimentares e estilo de vida da população como os principais fatores associados a este incremento, de acordo com a OMS. $_{(1,4)}$

O DM apresenta alta morbimortalidade e os pacientes tem perda importante na qualidade de vida, sendo esta considerada uma epidemia mundial acarretando alto custo tanto econômico como social. $\mathrm{O}$ número de pessoas vem aumentando nas mesmas proporções que o envelhecimento e aumento da prevalência de obesidade e sedentarismo ${ }_{(5)}$, sendo a educação em saúde uma das estratégias que pode contribuir para reduzir a alta prevalência de complicações além de prevenir a incidência da doença, o que se demonstra como um desafio para a equipe da estratégia de saúde da família (ESF).

O aumento da prevalência do diabetes, aliado à complexidade de seu tratamento, tais como restrições dietéticas, uso de medicamentos e complicações crônicas associadas (retinopatias, nefropatia, neuropatia, cardiopatia, pé neuropático, entre outras) reforçam a necessidade de programas educativos eficazes, individual e em grupo, adoção de dieta balanceada e da prática de atividades físicas,as quais são fundamentais para o tratamento em conjunto com terapia medicamentosa para redução das complicações ${ }_{(6)}$

Estudos têm demonstrado a necessidade de acompanhamento, de apoio e de seguimento contínuo do paciente diabético por equipe multiprofissional, de tal forma os pacientes acompanhados de forma sistemática, preveniram e/ou protelaram as complicações crônicas durante a evolução do diabetes mellitus. ${ }_{(7-11)}$

Por tanto se faz necessário a realização de grupos educativos envolvendo os pacientes diabéticos, para mudanças de estilos de vida relacionados com a dieta, pratica de atividades físicas, cumprimento do tratamento de forma supervisionada com atuação em conjunto da Equipe de ESF.

Logo, o presente estudo relata a experiência das ações educativas com pacientes diabéticos e de 
uma unidade de saúde inserida no Programa Mais Médicos, a partir da construção de um projeto de intervenção da médica inscrita neste programa, ao longo de 2 anos de sua existência, a partir da percepção desta profissional de que os indivíduos com diabetes apresentavam dificuldades em relaçãoao autocuidado.

\section{MÉTODO}

Trata-se de um relato de experiência baseado a partir da necessidade de construção de um projeto de intervenção como trabalho de conclusão da especialização do Programa Mais Médicos. Dessa forma , foi realizado gruposde educação em saúdesobre autocuidado com 50 pacientes diabéticos cadastrados na unidade de saúde, Ipuca São Fidelis ,Rio de Janeiro, com ações desenvolvidas por uma equipe multidisciplinar, com o objetivo de interferir de maneira positiva na linha de controle metabólico as pessoas com diagnostico de Diabetes Mellitus,identificando saberes dos pacientes diabéticos sobre a doença e seu tratamento em especial as formas de lidar com a doença, sendo orientados os pacientes quanto à complicações, cronicidade da diabetes Mellitus, formas de tratamento enfatizando a importância da influência do estilo de vida saudável, estimular a prática de exercícios físicos e dieta saudável.

\section{Relato do planejamento e realização das oficinas}

Inicialmente foi realizada uma reunião com todos os membros da equipe: enfermeiro, técnico de enfermagem, psicólogo, educador físico, para sensibilizá-los sobre o projeto, em especial os agentes comunitários de saúde (ACS), para que em suas visitas domiciliares, divulgassem as ofertas do serviço às famílias.

Na primeira etapa foi feita a identificação e convite dos pacientes diabéticos cadastrados na UBS por micro área para participação do projeto, durante as consultas individuais foram se monitorando o peso, a glicose capilar, pressão arterial e a conscientização de importância da consulta periódica, bem como, a prática de exercícios físicos e uma dieta saudável. Além desses grupos foram criadas visitas domiciliares rotineiras em pacientes que a equipe designou com casos mais complexos e que poderiam ser ajudados a melhorar o controle metabólico da doença.

Na seguinte etapa, foram realizadas reuniões quinzenais $\left(1^{\mathrm{a}}\right.$ e $3^{\mathrm{a}}$ terças-feiras de cada mês) na unidade para discussão dos temas abaixo.

1- Troca de experiências entre doentes crônicos e equipe para levantar os saberes dessa população com relação à Diabetes Mellitus (Equipe multidisciplinar); 2- A partir dos conhecimentos trazidos pelos pacientes, a equipe interdisciplinar fez uma abordagem sobre as complicações e a cronicidade da doença e suas formas de tratamento, enfatizando a importância das mudanças no estilo de vida; 3- Importância da prática de exercícios físicos no controle da Diabetes Mellitus. 4- Alterações psicossociais da Diabetes Mellitus; 5- Importância do apoio da família nas mudanças de estilos de vida. 


\section{RESULTADOS E DISCUSSÃO}

O tema da promoção da saúde e da prevenção de doenças foi colocado em pauta na Resolução $n^{\circ}$ 94/05, pela Agência Nacional de Saúde Suplementar (ANS), que é o órgão regulador deste seguimento e por meio de subsídios econômicos, tem incentivado as operadoras a desenvolver programas e práticas que abordam hábitos de vida saudáveis entre seus associados, em especial o $\mathrm{DM}_{(1-3)}$

O autocuidado pode ser definido como a prática de atividades que as pessoas realizam em seu próprio benefício na manutenção da vida, saúde e bem-estar e o desenvolvimento dessa prática está diretamente relacionado às habilidades, limitações, valores, regras culturais e científicas da própria $\operatorname{pessoa~}_{(3)^{\circ}}$.

Autocuidado significa deixar de ser passivo em relação aos cuidados e diretrizes apontadas pela medicina. Trata-se de um comportamento pessoal, que pode influenciar na saúde, porém não se dá de maneira isolada, mas em conjunto com fatores ambientais, sociais, econômicos, hereditários e relacionados aos serviços da saúde ${ }_{\text {(4) }}$

De maneira geral, os portadores de diabetes mellitus reconhecem a importância e a necessidade dos cuidados com os pés para evitar complicações, porém o autocuidado não é realizado corretamente Ressalta-se ainda que a presença de complicações pode diminuir a motivação para o autocuidado, frente às limitações relacionadas a elas

Considerado um dos principais componentes no tratamento do diabetes, o autocuidado envolve o segmento de um plano alimentar, a monitorização da glicemia capilar, a realização de atividades físicas, o uso correto da medicação e os cuidados com os pés.

A educação para o automanejo é o processo de ensinar o usuário a administrar a sua doença. As metas da educação em diabetes consistem em melhorar o controle metabólico, prevenir as complicações agudas e crônicas, e melhorar a qualidade de vida com custos razoáveis. No entanto, há déficit significativo de conhecimento e de habilidade em 50 a $80 \%$ dos indivíduos acometidos por esta doença. Durante o processo educativo, o usuário deve, em conjunto com a equipe multiprofissional de saúde, buscar estratégias efetivas que o auxiliem a manejar a doença. Esse é dos mais importantes investimentos em longo prazo que a sociedade pode oferecer, já que os custos da saúde dos indivíduos, desencadeados pelas complicações da doença, são enormes.

\section{CONCLUSÃO}

Aeducação para o autocuidadoé aspecto fundamental do tratamento à pessoa com diabetes mellitus e sua importância é reconhecida por os usuários com diferentes características socioeconômicos e culturais Para a educação efetiva em diabetes é necessário treinamento, conhecimento, habilidades pedagógicas, capacidade de comunicação e de escuta, compreensão e capacidade de negociação 
pela equipe multiprofissional de saúde

Observou-se que a partir das informações em saúde compartilhadas durante as oficinas com esse grupo de pacientes, de modo geral, contribuíram para que esse grupo tivessem uma percepção melhor sobre seu autocuidado, refletindo para alcançar um bom controle metabólico a partir da compreensão da doença e do manejo do tratamento, melhorarem o conhecimento sobre sua doença, seus cuidados que pudessem implicar em mudanças no estilo de vida, especialmente, a prática de exercícios físicos e realização de uma dieta balanceada, o que, consequentemente, fez diferença no tratamento da doença.

Os programas de educação em diabetes permitem a vivência e a experiência em se trabalhar de forma conjunta, integrando e compartilhando conhecimentos, visando a redução da mortalidade por esta doença e para melhorar a qualidade de vida e o melhor cuidado do usuário e da comunidade.

Aeducação para o autocuidado é aspecto fundamental do tratamento à pessoa com diabetes mellitus e sua importância é reconhecida por os usuários com diferentes características socioeconômica e culturais Para a educação efetiva em diabetes é necessário treinamento, conhecimento, habilidades pedagógicas, capacidade de comunicação e de escuta, compreensão e capacidade de negociação pela equipe multiprofissional de saúde.

O Programa Mais Médicos (PMM) é parte de um amplo esforço do Governo Federal, com apoio de estados e municípios, para a melhoria do atendimento aos usuários do Sistema Único de Saúde (SUS). O programa busca resolver a questão emergencial do atendimento básico ao cidadão, mas também cria condições para continuar a garantir um atendimento qualificado no futuro para aqueles que acessam cotidianamente o SUS. Além de estender o acesso, o programa provoca melhorias na qualidade e humaniza o atendimento, com médicos que criam vínculos com seus pacientes e com a comunidade.

\section{REFERÊNCIAS BIBLIOGRÁFICAS}

1- JC Ribeiro,JA Duarte .Diabetes mellitus: razão de prevalências nas diferentes regiões geográficas no Brasil, 2002 - 2007 Ciênc. saúde coletiva, 2012 - SciELO Brasil

2-Organização Mundial da Saúde - OMS. Cuidados inovadores para condições crônicas: componentes estruturais de ação: relatório mundial. Brasília (DF): OMS; 2003.

3- MJ Michels, MH Coral, TM Sakae, TB Damas ,LM Furlanetto . Questionário de atividades de autocuidado com diabetes: tradução, adaptação e avaliação das propriedades psicométricas. Arq Bras EndocrinolMetab. 2010;54(7):644-50.

4- Gomides D, Gomes LC, Martins AC, Pac AE. Autocuidado das pessoas com diabetes mellitus que possuem complicações em membros inferiores. Acta Paul Enferm. 2013; 26(3):289- 
93.

5-Gimenes HT, Veras VS, Da Franca AX, De Souza CT, Zanetti ML, Dos Santos MA.Qualidade de vida de pacientes com diabetes mellitus antes e após participação em programa educativo. Rev Esc Enferm USP, 2013 - SciELOBrasil .

6-Livro RA Barsaglini - 2011 - books.google.com .As representações sociais e a experiência com o diabetes: um enfoque socioantropológico

7- SN Cordeiro, RM Júnior... Significados da dieta e mudanças de hábitos para portadores de doenças metabólicas crônicas: Ciênc. saúde coletiva, 2011 - SciELO Brasil

8- DC Malta, JB Silva Jr - Epidemiologia e Serviços de Saúde, 2013 - scielo.iec.pa.gov.br

9- MR Viana, TT Rodriguez -Complicação cardiovasculares e renais no diabetes mellitus. Revista de Ciências Médicas e ..., 2012 - portalseer.ufba.br

10- Efeito da ação educativa sobre o conhecimento da doença e o controle metabólico de pacientes com diabetes mellitus tipo 2 Da Pereira - 2011 - repositorio.bc.ufg.br http://repositorio. bc.ufg.br/tede/handle/tde/1472

11- LF Frigo, RM da Silva, KM de Mattos... - ... de Epidemiologia e ..., 2013 - online.unisc. br. Ação educativa interdisciplinar para pacientes com diabetes na atenção básica: uma revisão bibliográfica

Artigo apresentado em 09-08-15

Artigo aprovado em 21-10-15 Artigo publicado no sistema em 30-12-15 